\title{
Dynamic Characteristics of Microring Driven by the Symmetrically Distributed Electrostatic Force
}

\author{
Qingheng Meng, ${ }^{1}$ Yuanlin Zhang, ${ }^{1}$ Jin Wei, ${ }^{1}$ Yuh-Chung Hu, ${ }^{2}$ Yan Shi, ${ }^{3}$ and Tao Yu $\mathbb{D}^{1}$ \\ ${ }^{1}$ School of Mechatronics and Automobile Engineering, Yantai University, Yantai 264005, China \\ ${ }^{2}$ Department of Mechanical and Electromechanical Engineering, National ILan University, Yilan, Yilan County 260, Taiwan \\ ${ }^{3}$ School of Automation Science and Electrical Engineering, Beihang University, Beijing 100191, China
}

Correspondence should be addressed to Tao Yu; yutao@ytu.edu.cn

Received 15 July 2020; Revised 12 October 2020; Accepted 21 January 2021; Published 4 February 2021

Academic Editor: Juan Sandoval

Copyright (c) 2021 Qingheng Meng et al. This is an open access article distributed under the Creative Commons Attribution License, which permits unrestricted use, distribution, and reproduction in any medium, provided the original work is properly cited.

\begin{abstract}
This paper aims at investigating the dynamic characteristics of a microring driven by dual arch electrodes because they are basic elements of microelectrostatic motors. The dual arch electrodes surround the periphery of the microring and are arranged symmetrically to the center of the ring. The electrodes are fixed while the microring is flexible. The electrostatic force will deform the microring, while the deflection of the microring changes the gap between the microring and the electrodes, thereby changing the electrostatic force. Therefore, this is an electromechanical coupling effect. The nonlinear partial-differential equation that governs the motion of the microring is derived based on thin shell theory. Then, based on the assumption of small deflection, the nonlinear governing equation is linearized by truncating the higher-order terms of the Taylor series expansion of the nonlinear electrostatic force. After that, the linearized governing equation is discretized into a set of ordinary differential equations using Galerkin method in which the mode shape functions of the ring are adopted. The influences of the structural damping of the microring and the span of the arch electrodes on the forced response and dynamical stabilities of the microring are investigated. The results show that the damping ratio has a great influence on the system instability during high-frequency excitation. The unstable region of the system can increase with the increase of the electrode span; the response amplitude can also be increased within a certain range.
\end{abstract}

\section{Introduction}

In microelectromechanical systems (MEMS), electrostatic principle is widely used in microsensors/actuators because of its advantages of easy control, fast response, low power consumption, and easy integration with IC processes [1]. Electrostatic forces are mainly used in gyroscopes [2], electrostatic motors [3], torsion micromirrors [4], accelerometers [5], and microswitches [6]. Their key microstructures include beams, plates, and rings. In the design of electrostatic driven microstructures, the energy domain coupling of electrical and mechanical energies is a very important issue. Vibration analysis of microstructures based on different composite materials is the focus of current research [7-10]. Vibration analysis of microstructures is different from macrostructures because the force proportional to the area plays a leading role [11]. The interaction of energy domains makes the analysis of MEMS complicated. Electrostatically driven microstructures will bring many nonlinear characteristics, such as dynamic instability, jumping, bifurcation, and chaos $[12,13]$. Stability is the basic condition for the system to work. An unstable system does not have the ability to adjust and cannot work normally $[14,15]$.

Since the 21st century, the research contents of electrostatic force in MEMS mainly include pull-in voltage, damping, stability, and amplitude frequency-response. For electrostatic driven devices, the driving voltage has a critical value, and the electrostatic force exhibits a strong nonlinearity at this value. When the driving voltage exceeds 
this critical value, the equilibrium between the elastic restoring force and the electrostatic force is broken, the microstructure and electrode are attracted together, and the pull-in phenomenon occurs. The voltage limit for the pull-in phenomenon is the pull-in voltage, and the corresponding position is called the pull-in position $[16,17]$. Farokhi et al. [18] studied the pull-in characteristics of electrostatically driven microarches and the effect of system parameters on the pull-in instability. The electrostatic switch needs to determine the pull-in voltage to prevent excessive voltage from causing adhesion failure of the structure [19]. The driving principle of the micromotor includes electrostatic type, electromagnetic type, and piezoelectric type [20-22]. In MEMS, since the existence of microscale effects, damping has a great impact on the function of microstructures, so damping cannot be ignored. Bao and Yang [23] surveyed kinds of literature on various damping models of MEMS microstructures and introduced related experiments. Belardinelli et al. [24] established a microbeam mechanical model considering both pressure film damping and thermoelastic damping and analyzed the effects of the two types of damping on the natural frequency of the beam. In the past, the research objects of electrostatically driven microstructures were mainly microplates and microbeams. Recently, researchers have begun to focus on other microstructures, such as the key components in gyroscopes and electrostatic motors: rings [25-28]. In the last century, researchers have established different theoretical models and systematically studied the inherent characteristics of the ring and the forced response under harmonic excitation [29]. Xu and Qin [30] theoretically studied the jump up and down phenomenon of electrostatically driven microring near the natural frequency and explored the effect of excitation voltage on the critical frequency of the jump phenomenon.

Dynamic pull-in instability needs to consider factors such as damping, AC voltage excitation, and inertial effects caused by sudden changes in DC voltage, which all affect the dynamic pull-in process [26]. In addition to studying the dynamic pull-in instability of the system, the stability of the system also needs to be considered. Hu et al. [31] studied the stability of electrostatic driven microbeam system theoretically. The authors of $[32,33]$ studied the stability and forced response of the microring system under the traveling electrostatic force, which is generated by an electrode. Yu et al. [34] established a theoretical model of a rotating ring under a uniform electric field to analyze the inherent characteristics and dynamic stability of the system. In the above study of the stationary ring, the electric field is generated by a constant voltage, without considering the effect of dynamic voltage. The microring is driven by pairs of electrodes. In the study of electrostatically driven microring, damping is mostly ignored, but damping has a great influence on the response and stability of the system. Therefore, in this paper, the physical model of a pair of electrostatically driven microrings under alternating voltage control is established, and the effect of damping on the system is considered.
The magnitude of the electrostatic force is proportional to the square of the voltage and inversely proportional to the square of the gap between the ring and the electrode. Based on the theory of thin shells and the electrostatic force model, a dynamic model of electrostatically driven microring is established $[29,35]$. The electrostatic force is controlled by a pair of fixed electrodes applying an AC voltage to control the deformation of the microring. This model takes into account the structural deformation coupling. Based on the small deflection assumption, the electrostatic force can be expanded using Taylor series to obtain a linear periodic timevarying system equation. Discrete partial differential equations use Galerkin method to obtain ordinary differential matrix equations. First, the system performs modal analysis, then Floquet theory is used to judge the stability of the system, and the forced response of the system is obtained by the RungeKutta numerical integration method. This paper studies the stability and the amplitude-frequency response of the microring, which provides a reference for the design of the distributed electrostatic force-driven microring.

\section{Model}

The main components of an electrostatic motor consist of a ring-shaped rotor and several pairs of arch-shaped driving electrodes. The driving electrodes surround the periphery of the ring at equal intervals and symmetrically to the center of the ring. Each pair of the driving electrode consists of two driving electrodes symmetrical to the center of the ring. When the motor is working, the voltage is sequentially applied to each pair of the driving electrode, and the ring is elastically deformed at the corresponding position. The periodic voltage generates a periodic electric field, which generates a periodic electrostatic force. The periodic deformation caused by the electrostatic force changes the capacitance between the electrode and the ring, thereby driving the ring to move [30].

Based on the aforesaid working principle, it is worthwhile to investigate the dynamic characteristics of the ring under the action of a pair of electrodes. As shown in Figure 1, a dynamic voltage is applied between the microring and the electrode, which generates a time-varying electric field to drive the ring. The potential difference $\widehat{V}$ is the control voltage, which is set to $\widehat{V}=\widehat{V}_{D}+\widehat{V}_{A} \sin (\widehat{\Omega} \hat{t})$, where $\widehat{V}_{D}, \widehat{V}_{A}, \widehat{\Omega}$, and $\widehat{t}$ are DC voltage, AC voltage, AC angular frequency, and time, respectively. The average radius and thickness of the ring are $r$ and $h$, the span of the two electrodes is $\phi$, and the gap between the ring and the electrode is $g$. There is a fixed coordinate system $X-Y$ on the ring, and the radial displacement caused by electrostatic force at the angular coordinate $\theta$ is $\widehat{u}(\theta, \widehat{t})$.

2.1. Equation of Motion. According to the principle of parallel capacitor plates, the distributed force shown in Figure 1 can be expressed as 


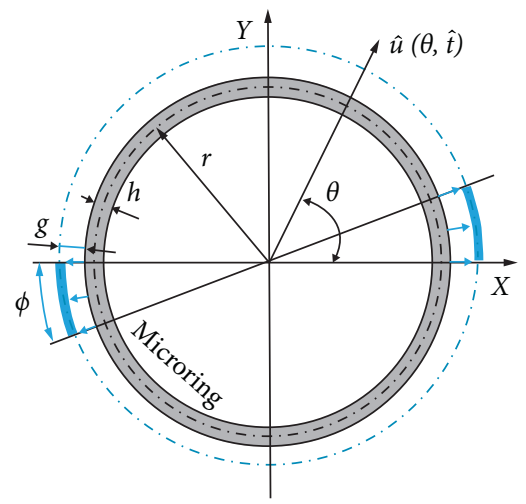

Figure 1: Schematic diagram of electrostatically driven microring.

$$
[H(\theta)-H(\theta-\phi)+H(\theta-\pi)-H(\theta-\pi-\phi)] \frac{\varepsilon b \widehat{V}^{2}}{2(g-\widehat{u})^{2}},
$$

where $\varepsilon$ is the permittivity of the medium between the ring and electrode, $b$ is the width of the ring, and $H$ represents the unit step function.

The microring of this system is mainly subjected to radial forces, so its circumferential inertial force is negligible. According to the thin shell theory [29], the dynamic equation of the ring subjected to the electrostatic force of Figure 1 is

$$
\begin{aligned}
\frac{E I}{r^{4}}\left(\frac{\partial^{4} \widehat{u}}{\partial \theta^{4}}+2 \frac{\partial^{2} \widehat{u}}{\partial \theta^{2}}+\widehat{u}\right)+\rho A \frac{\partial^{2} \widehat{u}}{\partial \hat{t}^{2}}= & {[H(\theta)-H(\theta-\phi)+H(\theta-\pi)} \\
& -H(\theta-\pi-\phi)] \frac{\varepsilon b \widehat{V}^{2}}{2(g-\widehat{u})^{2}} .
\end{aligned}
$$

For easier essay writing, $H(\theta)-H(\theta-\phi)+H(\theta-\pi)-$ $H(\theta-\pi-\phi)$ is set to $f(\theta, \phi)$. The authors introduce dimensionless variables as

$$
\begin{gathered}
u=\widehat{u} / g, t=\widehat{t} / T, \Omega=\widehat{\Omega} T, V_{D}=\widehat{V}_{D} \sqrt{\frac{r^{3} \varepsilon b}{4 \phi E I g^{3}}}, \\
V_{A}=\widehat{V}_{A} \sqrt{\frac{r^{3} \varepsilon b}{4 \phi E I g^{3}}}, V=V_{D}+V_{A} \sin (\Omega t),
\end{gathered}
$$

where $T=\sqrt{\rho A r^{4} / E I}$. Using the dimensionless variables in Equation (3), the dimensionless motion equation is expressed as

$$
\frac{\partial^{4} u}{\partial \theta^{4}}+2 \frac{\partial^{2} u}{\partial \theta^{2}}+u+\frac{\partial^{2} u}{\partial t^{2}}=f(\theta, \phi) \frac{V^{2}}{(1-u)^{2}} .
$$

Based on the assumption of small deformation, the nonlinear part of the electrostatic force term is expanded using the Taylor series concerning the initial equilibrium position $(u=0)$, ignoring the higher-order terms and retaining the linear terms, that is

$$
\frac{1}{(1-u)^{2}} \approx 1+2 u \text {. }
$$

Finally, a linear time-varying motion equation is obtained as

$$
\frac{\partial^{2} u}{\partial t^{2}}+\frac{\partial^{4} u}{\partial \theta^{4}}+2 \frac{\partial^{2} u}{\partial \theta^{2}}+\left(1-2 f(\theta, \phi) V^{2}\right) u=f(\theta, \varphi) V^{2} .
$$

Due to the alternating voltage on the left side of the equation of motion (6), the microring system becomes a time-varying system, and the driving voltage $V$ can change the dynamic characteristics of the microring.

2.2. Discretization. For the geometrical periodicity of a circular ring, the deflection function $u(\theta, t)$ can be represented by

$$
u(\theta, t)=\sum_{k=2}^{n}\left[\alpha_{k}(t) \cos k \theta+\beta_{k}(t) \sin k \theta\right]
$$

where $k$ is the circumferential wave number and $\alpha_{k}(t)$ and $\beta_{k}(t)$ are generalized coordinate functions about time $t$. The term $k=1$ is a rigid body mode, so it can be eliminated $[29,34]$, such that $k=2,3 \ldots n$ form a complete basis space. Substituting equation (7) in equation (6) gives 


$$
\begin{aligned}
& \sum_{k=2}^{n}\left[\ddot{\alpha}_{k}(t)+k^{4} \alpha_{k}(t)-2 k^{2} \alpha_{k}(t)+\left[1-2 f(\theta, \phi) V^{2}\right] \alpha_{k}(t)\right] \cos k \theta \\
& \quad+\sum_{k=2}^{n}\left[\ddot{\beta}_{k}(t)+k^{4} \alpha_{k}(t)-2 k^{2} \beta_{k}(t)+\left[1-2 f(\theta, \phi) V^{2}\right] \beta_{k}(t)\right] \sin k \theta \\
& =f(\theta, \phi) V^{2} .
\end{aligned}
$$

Since the orthogonality of trigonometric functions, one can discretize the equation (8) by multiplying equation (8) with $\cos (m \theta)$ and $\sin (m \theta))$, where $m=2,3,4, \ldots$, which gets the following two equations:

$$
\begin{aligned}
& \sum_{k=2}^{n}\left[\ddot{\alpha}_{k}(t)+k^{4} \alpha_{k}(t)-2 k^{2} \alpha_{k}(t)+\left[1-2 f(\theta, \phi) V^{2}\right] \alpha_{k}(t)\right] \cos k \theta \cos (m \theta) \\
& \quad+\sum_{k=2}^{n}\left[\ddot{\beta}_{k}(t)+k^{4} \alpha_{k}(t)-2 k^{2} \beta_{k}(t)+\left[1-2 f(\theta, \phi) V^{2}\right] \beta_{k}(t)\right] \sin k \theta \cos (m \theta) \\
& =f(\theta, \phi) V^{2} \cos (m \theta), \\
& \sum_{k=2}^{n}\left[\ddot{\alpha}_{k}(t)+k^{4} \alpha_{k}(t)-2 k^{2} \alpha_{k}(t)+\left[1-2 f(\theta) V^{2}\right] \alpha_{k}(t)\right] \cos k \theta \sin (m \theta) \\
& \quad+\sum_{k=2}^{n}\left[\ddot{\beta}_{k}(t)+k^{4} \alpha_{k}(t)-2 k^{2} \beta_{k}(t)+\left[1-2 f(\theta) V^{2}\right] \beta_{k}(t)\right] \sin k \theta \sin (m \theta) \\
& =f(\theta) V^{2} \sin (m \theta) .
\end{aligned}
$$

Integrating equations (9) and (10) over the microring's circumference gives

$$
\begin{aligned}
& \sum_{k=2}^{n}\left[\ddot{\alpha}_{k}(t)+\left(k^{2}-1\right)^{2} \alpha_{k}(t)\right] \int_{0}^{2 \pi} \cos k \theta \cos (m \theta) d \theta-\sum_{k=2}^{n} 2 V^{2} \alpha_{k}(t) \int_{0}^{2 \pi} f(\theta, \phi) \cos k \theta \cos (m \theta) d \theta \\
& \quad+\sum_{k=2}^{n}\left[\ddot{\beta}_{k}(t)+\left(k^{2}-1\right)^{2} \beta_{k}(t)\right] \int_{0}^{2 \pi} \sin k \theta \cos (m \theta) d \theta-\sum_{k=2}^{n} 2 V^{2} \beta_{k}(t) \int_{0}^{2 \pi} f(\theta, \phi) \sin k \theta \cos (m \theta) d \theta \\
& =V^{2} \int_{0}^{2 \pi} f(\theta, \phi) \cos (m \theta) d \theta, \\
& \sum_{k=2}^{n}\left[\ddot{\alpha}_{k}(t)+\left(k^{2}-1\right)^{2} \alpha_{k}(t)\right] \int_{0}^{2 \pi} \cos k \theta \sin (m \theta) \mathrm{d} \theta-\sum_{k=2}^{n} 2 V^{2} \alpha_{k}(t) \int_{0}^{2 \pi} f(\theta, \phi) \cos k \theta \sin (m \theta) \mathrm{d} \theta \\
& \quad+\sum_{k=2}^{n}\left[\ddot{\beta}_{k}(t)+\left(k^{2}-1\right)^{2} \beta_{k}(t)\right] \int_{0}^{2 \pi} \sin k \theta \sin (m \theta) \mathrm{d} \theta-\sum_{k=2}^{n} 2 V^{2} \beta_{k}(t) \int_{0}^{2 \pi} f(\theta, \phi) \sin k \theta \sin (m \theta) \mathrm{d} \theta \\
& =V^{2} \int_{0}^{2 \pi} f(\theta, \phi) \sin (m \theta) \mathrm{d} \theta .
\end{aligned}
$$


Due to the orthogonality of trigonometric functions, Equations (11) and (12) can be simplified and expressed in the following matrix expression:

$$
\{\ddot{X}\}_{2(n-1) \times 1}+[\mathbf{K}]_{2(n-1) \times 2(n-1)}\{\mathbf{X}\}_{2(n-1) \times 1}=\{\mathbf{Q}\}_{2(n-1) \times 1},
$$

where $\{\mathbf{X}\},[\mathbf{K}]$, and $\{\mathbf{Q}\}$ are generalized coordinates vector, stiffness matrix, and generalized force vector, respectively. They are specifically expressed as

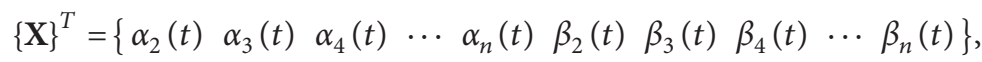

$$
\begin{aligned}
& {[\mathbf{K}]=\left[\mathbf{K}^{k}\right]_{2(n-1) \times 2(n-1)}+\left[\mathbf{K}^{e}\right]_{2(n-1) \times 2(n-1)},}
\end{aligned}
$$

where the stiffness matrix $[\mathbf{K}]$ consists of $\left[\mathbf{K}^{k}\right]$ and $\left[\mathbf{K}^{e}\right]$. $\left[\mathbf{K}^{k}\right]$ is structural stiffness-matrix, which is a diagonal matrix. $\left[\mathbf{K}^{e}\right]$ is electrostatic stiffness-matrix due to the dynamic voltage. The elements in the stiffness matrix are expressed as

$$
\begin{aligned}
& \left\{\begin{array}{l}
K_{i j}^{k}=\left(i^{2}-1\right)^{2}, \quad(i=j), \\
K_{i j}^{k}=0, \quad(i \neq j)
\end{array}\right. \\
& {\left[\mathbf{K}^{e}\right]=\left[\begin{array}{ll}
{\left[\mathbf{K}^{a}\right]_{(n-1) \times(n-1)}} & {\left[\mathbf{K}^{b}\right]_{(n-1) \times(n-1)}} \\
{\left[\mathbf{K}^{c}\right]_{(n-1) \times(n-1)}} & {\left[\mathbf{K}^{d}\right]_{(n-1) \times(n-1)}}
\end{array}\right],} \\
& K_{i j}^{a}=\left\{\begin{array}{l}
-\frac{V^{2}}{\pi}\left[\frac{\sin (i+j) \phi}{i+j}\left(1+(-1)^{i+j}\right)+\frac{\sin (i-j) \phi}{i-j}\left(1+(-1)^{i-j}\right)\right], \\
-\frac{2 V^{2}}{\pi}\left[\phi+\frac{\sin (2 i \phi)}{4 i}+\frac{\sin (2 i(\pi+\phi))}{4 i}\right], \quad i=j,
\end{array}\right. \\
& K_{i j}^{b}=\left\{\begin{array}{l}
\frac{V^{2}}{\pi}\left[\frac{\cos (i+i) \phi}{i+j}\left(1+(-1)^{i+j}\right)+\frac{\cos (i-j) \phi}{i-j}\left(1+(-1)^{i-j}\right)-\frac{2 i}{i^{2}-j^{2}}-\left[\frac{(-1)^{i+j}}{i+j}+\frac{(-1)^{i-j}}{i-j}\right]\right], \\
-\frac{2 V^{2}}{\pi}\left[\frac{(\sin i \phi)^{2}}{2 i}+\frac{(\sin i(\pi+\phi))^{2}}{2 i}\right], \quad i=j,
\end{array}\right. \\
& K_{i j}^{c}=\left\{\begin{array}{l}
\frac{V^{2}}{\pi}\left[\frac{\cos (i+j) \phi}{i+j}\left(1+(-1)^{i+j}\right)-\frac{\cos (i-j) \phi}{i-j}\left(1+(-1)^{i-j}\right)+\frac{2 j}{i^{2}-j^{2}}-\left[\frac{(-1)^{i+j}}{i+j}-\frac{(-1)^{i-j}}{i-j}\right]\right], \\
-\frac{2 V^{2}}{\pi}\left[\frac{(\sin k \phi)^{2}}{2 k}+\frac{(\sin k(\pi+\phi))^{2}}{2 k}\right], \quad i=j .
\end{array}\right. \\
& K_{i j}^{d}=\left\{\begin{array}{l}
\frac{V^{2}}{\pi}\left[\frac{\sin (i+j) \phi}{i+j}\left(1+(-1)^{i+j}\right)-\frac{\sin (i-j) \phi}{i-j}\left(1+(-1)^{i-j}\right)\right], \\
-\frac{2 V^{2}}{\pi}\left[\phi-\frac{\sin 2 k \phi}{4 k}-\frac{\sin 2 k(\pi+\phi)}{4 k}\right], \quad i=j,
\end{array}\right.
\end{aligned}
$$

The generalized force vector is 


$$
\begin{aligned}
& \{\mathbf{Q}\}=\left\{\begin{array}{l}
\left\{\mathbf{Q}^{\alpha}\right\}_{(n-1) \times 1} \\
\left\{\mathbf{Q}^{\beta}\right\}_{(n-1) \times 1}
\end{array}\right\}, \\
& \left\{\begin{array}{l}
Q_{i}^{\alpha}=\frac{V^{2}}{\pi}\left(\frac{\sin (i \phi)}{i}+\frac{\sin (i(\pi+\phi))}{i}\right), \\
Q_{i}^{\beta}=\frac{V^{2}}{\pi}\left(-\frac{\cos (i \phi)}{i}+\frac{1}{i}-\frac{\cos (i(\pi+\phi))}{i}+\frac{\cos (i \pi)}{i}\right) .
\end{array}\right.
\end{aligned}
$$

2.3. Damping. In this microring system, there is more or less a certain amount of damping, and it is generally obtained from experiments. The effect of damping on the forced
TABLE 1: Material parameters and geometric parameters.

\begin{tabular}{lc}
\hline Properties & value \\
\hline Density & $\rho=2.7 \times 10^{3} \mathrm{Kg} / \mathrm{m}^{3}$ \\
Young's modulus & $E=6.8 \times 10^{10} \mathrm{~Pa}$ \\
Mean radius & $r=0.02 \mathrm{~m}$ \\
Radial thickness & $h=3 \times 10^{-4} \mathrm{~m}$ \\
Gap & $g=6 \times 10^{-5} \mathrm{~m}$ \\
Width & $b=5 \times 10^{-3} \mathrm{~m}$ \\
\hline
\end{tabular}

response is to eliminate the transient response and eventually reach the steady state. In practical applications, the steady-state response is the focus of research. Adding equivalent damping in the radial direction of the ring is to add the damping term $\lambda \dot{u}$ to equation (4). According to previous studies, the damping matrix can be set to $[29,36]$

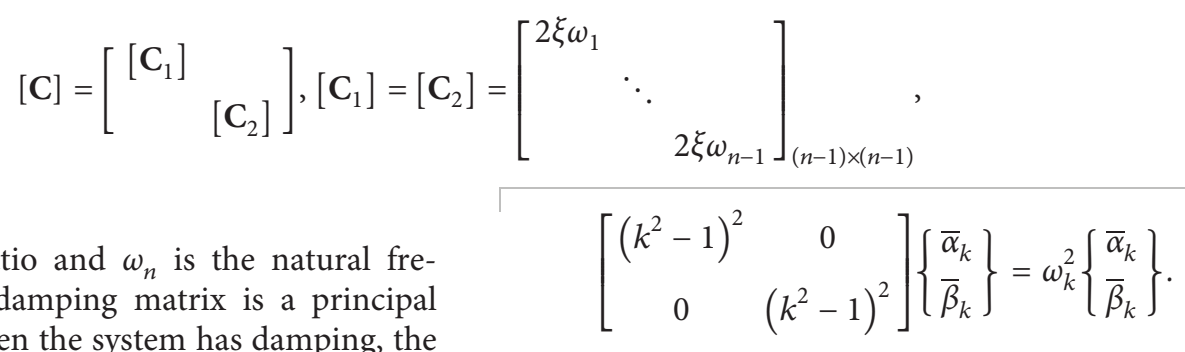

Generally, the amplitudes $\bar{\alpha}_{k}$ and $\bar{\beta}_{k}$ are not zero, and the natural frequency can be obtained, namely, $\omega_{k-1}^{2}=\left(k^{2}-1\right)^{2}$, $(k=2,3,4, \ldots)$. The corresponding natural modes of the microring are $\cos k \theta+\sin k \theta, \quad(k=2,3,4, \ldots)$. Figure 2 shows the first three natural modes of the microring.

\section{Dynamic Analysis}

In this section, the mode of the microring is derived. Table 1 shows the material parameters and geometric parameters of the microring.

Modal analysis is to find the inherent characteristics of the system when there is no external force, such as natural frequency and mode shape. When the system has no electrostatic force and the damping is neglected, equation (24) can be simplified to

$$
\left\{\begin{array}{l}
\ddot{\alpha}_{k}(t) \\
\ddot{\beta}_{k}(t)
\end{array}\right\}+\left[\begin{array}{cc}
\left(k^{2}-1\right)^{2} & 0 \\
0 & \left(k^{2}-1\right)^{2}
\end{array}\right]\left\{\begin{array}{l}
\alpha_{k}(t) \\
\beta_{k}(t)
\end{array}\right\}=\left\{\begin{array}{l}
0 \\
0
\end{array}\right\},
$$

where $k=2,3,4 \cdots$. The generalized coordinate functions $\alpha_{k}(t)$ and $\beta_{k}(t)$ are usually set to

$$
\left\{\begin{array}{l}
\alpha_{k}(t) \\
\beta_{k}(t)
\end{array}\right\}=\left\{\begin{array}{l}
\bar{\alpha}_{k} \\
\bar{\beta}_{k}
\end{array}\right\} e^{i \omega_{k} t},
$$

4.1. Forced Response. The differential equations (24) are numerically solved using the Runge-Kutta method. It is necessary to reduce the order of the second-order differential equations and transform them into the state space. The space state vector is defined as

$$
\{\mathbf{Y}\}_{4(n-1) \times 1}=\left\{\begin{array}{l}
\{\mathbf{X}\} \\
\{\dot{\mathbf{X}}\}
\end{array}\right\} .
$$

Equation (24) is transformed into the state space and expressed as

$$
\{\dot{Y}\}=[\mathbf{A}]\{\mathbf{Y}\}+[\mathbf{B}]\{\mathbf{F}\}
$$

where

$$
\begin{aligned}
& {[\mathbf{A}]_{4(n-1) \times 4(n-1)}=\left[\begin{array}{cc}
{[0]_{2(n-1) \times 2(n-1)}} & {[\mathbf{I}]_{2(n-1) \times 2(n-1)}} \\
-[\mathbf{K}]_{2(n-1) \times 2(n-1)} & -[\mathbf{C}]_{2(n-1) \times 2(n-1)}
\end{array}\right],} \\
& {[\mathbf{B}]_{4(n-1) \times 4(n-1)}=\left[\begin{array}{cc}
{[0]_{2(n-1) \times 2(n-1)}} & {[0]_{2(n-1) \times 2(n-1)}} \\
{[0]_{2(n-1) \times 2(n-1)}} & {[\mathbf{I}]_{2(n-1) \times 2(n-1)}}
\end{array}\right],}
\end{aligned}
$$
respectively. Inserting equation (26) into equation (25) yields the following matrix expression: 


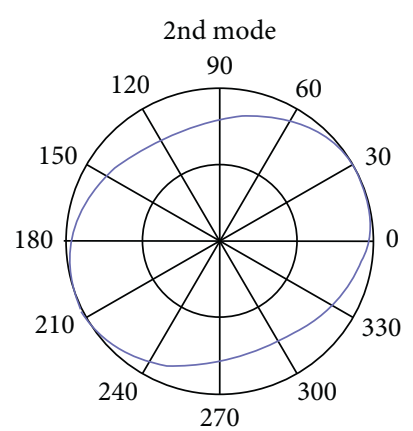

(a)

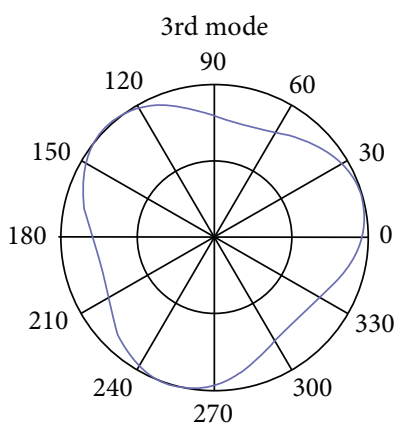

(b)

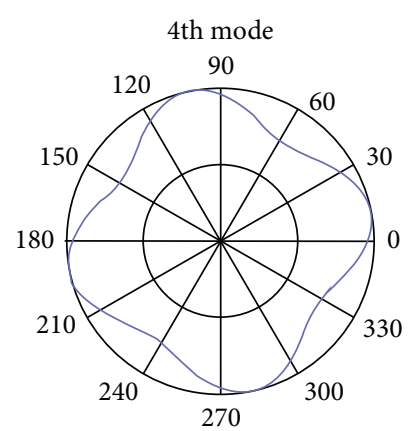

(c)

FIGURE 2: The first three natural modes.

$$
\{\mathbf{F}\}_{4(n-1) \times 1}=\left\{\begin{array}{l}
\{\mathbf{Q}\}_{2(n-1) \times 1} \\
\{\mathbf{Q}\}_{2(n-1) \times 1}
\end{array}\right\},
$$

where [0] and $[\mathbf{I}]$ are the zero matrix and the unit matrix, respectively. Since the $[\mathbf{A}]$ matrix contains $[\mathbf{K}]$, the matrix is periodically time-varying.

4.2. Stability. According to the static stiffness terms (17)-(21), the stiffness term of this system is related to the voltage, and the voltage changes periodically with time, so the system is a periodic time-varying system. The stability of periodic time-varying systems is an important subject. According to the characteristics of the system, Floquet theory is used to analyze its stability.

To analyze the stability of the system, the homogeneous part of equation (29) needs to be considered, that is,

$$
\{\dot{Y}\}=[\mathbf{A}]\{\mathbf{Y}\} .
$$

The parameter matrix $[\mathbf{A}]$ is a periodically time-varying matrix with the period of $\widehat{T}=2 \pi /(2 \Omega)$, and set $4(n-1)$ linearly independent initial conditions:

$$
\{\mathbf{Y}(0)\}_{1}=\left\{\begin{array}{c}
1 \\
0 \\
0 \\
\vdots \\
0
\end{array}\right\},\{\mathbf{Y}(0)\}_{2}=\left\{\begin{array}{c}
0 \\
1 \\
0 \\
\vdots \\
0
\end{array}\right\}, \cdots,\{\mathbf{Y}(0)\}_{4(n-1)}=\left\{\begin{array}{c}
0 \\
0 \\
0 \\
\vdots \\
1
\end{array}\right\}
$$

The solution of equation (33) in a period $\widehat{T}$ is solved by the RungeKutta numerical method, namely, $\{\mathbf{Y}(\widehat{T})\}_{1},\{\mathbf{Y}(\widehat{T})\}_{2}, \ldots,\{\mathbf{Y}(\widehat{T})\}_{4(n-1)}$, and the solutions of these homogeneous equations are formed into a matrix [D]:

$$
[\mathbf{D}]=\left[\{\mathbf{Y}(\widehat{T})\}_{1},\{\mathbf{Y}(\widehat{T})\}_{2}, \ldots,\{\mathbf{Y}(\widehat{T})\}_{4(n-1)}\right] .
$$

The stability of the system can be determined by the eigenvalues $\left(\mu_{i}\right)$ of matrix [D] [37]. The system is stable if all the eigenvalues have magnitudes less than unity, i.e., $\left|\mu_{i}\right|<1$, unstable if at least one eigenvalue greater than unity, i.e., $\left|\mu_{i}\right|>1$, and marginally stable if at least one eigenvalue with unit magnitude and multiplicity less than unity.

\section{Results and Discussion}

To investigate the dynamic characteristics of the electrostatically driven microring system, the stability of the system is first analyzed, and then the amplitude-frequency response curve of the system is solved. The following stability analysis and forced response analysis are developed using the first three modes. Considering that the microring is driven by three pairs of electrodes, the maximum electrode span is $\pi / 3$, so the electrode span range is $[0, \pi / 3]$. The authors selected the angular coordinate $\theta=0$ for dynamic characteristics analysis.

5.1. Unstable Region. Damping is a factor that cannot be ignored in the system. Here, we first consider the impact of damping on system stability. Using the stability analysis method in Section 4.2, the unstable region of the system under different damping is studied.

Figure 3 shows the unstable region of the system when the AC voltage $V_{d}=0.5$ and the electrode span $\phi=\pi / 3$. The four figures show the unstable regions of the system when the damping ratios $\xi=0.0001,0.001,0.01$, and 0.1 , respectively. It can be seen that the unstable region mainly appears at the natural frequency and $2 \omega_{1}, 3 \omega_{1}, \omega_{1} / 2$, and $2 \omega_{1} / 3$. When the damping ratio is less than 0.01 , the system is most likely to show instability at $\omega_{1}$ and $2 \omega_{1}$. When the damping ratio is equal to 0.1 , the system is most likely to show instability at 


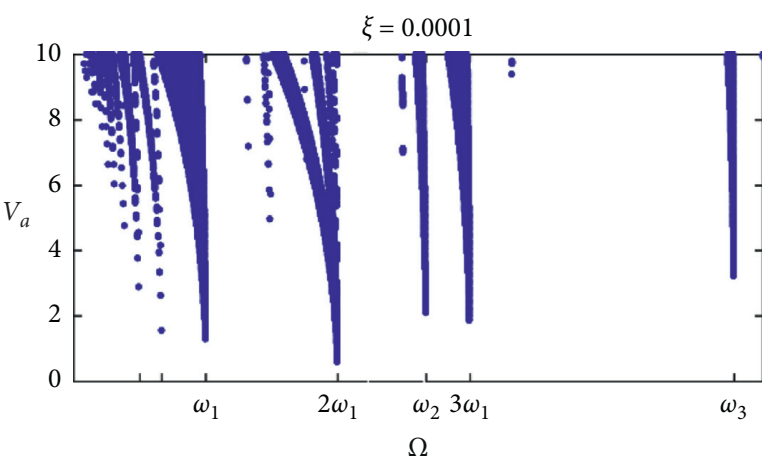

(a)

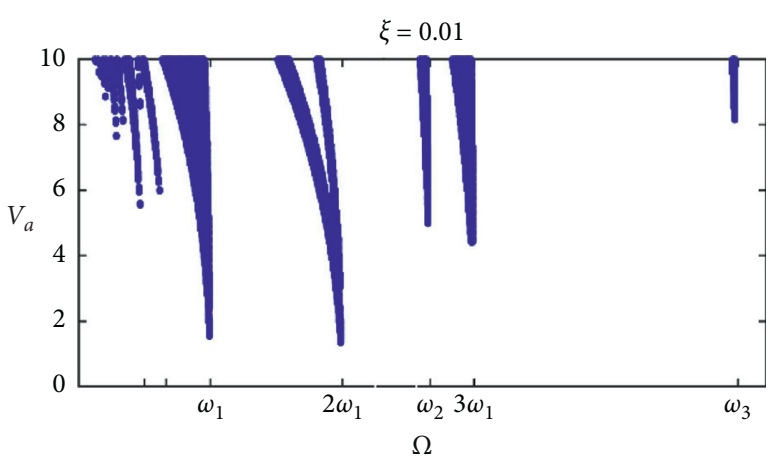

(c)

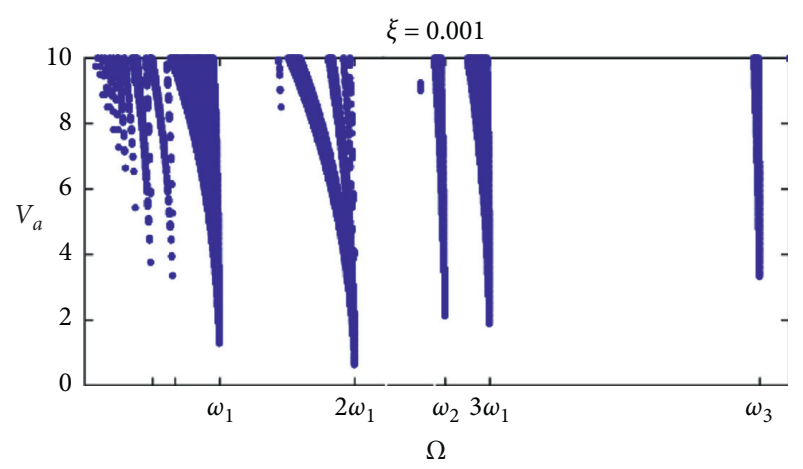

(b)

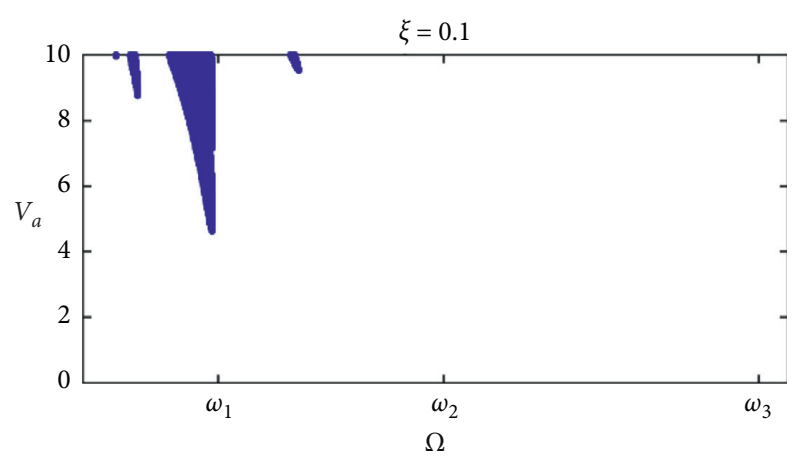

(d)

Figure 3: The stabilities of the circular-ring with respect to AC voltage and voltage frequency $\Omega$ for different damping ratio. $\xi$.

$\Omega=\omega_{1}$. The system easily exhibits instability at the firstorder natural frequency. When the damping ratio changes from 0.0001 to 0.001 , the unstable region of the system changes little. When the damping is increased, the change in the unstable region at high frequencies is relatively large. When the damping ratio is equal to 0.1 , the unstable region near the first-order natural frequency is most obvious.

The electrode span $\phi$ also affects the unstable region of the system. Figure 4 shows the unstable region of the system under different electrode span when the damping ratio $\xi=0.001$. It can be seen that increasing the electrode span increases the width of the unstable region and also decreases the minimum voltage value of the unstable region.

From Figure 5, one noticed that the relationship between the minimum voltage value of the unstable region and the electrode angle when the excitation frequencies are $\omega_{1}$ and $2 \omega_{1}$. As shown in Figure 5, increasing the angle of the electrode decreases the minimum voltage value in the unstable region. Combined with Figure 4, it can be seen that increasing the electrode span increases the unstable region. When the electrode angle is small, the minimum voltage value $V_{\min }$ will decrease rapidly as the electrode span $\phi$ increases. As the electrode span increases, the change rate of the voltage value becomes slower. When the electrode span is close to $\pi / 3$, the change rate of the lowest voltage is close to zero.
5.2. Amplitude-frequency Response. The equation (29) is solved under zero initial conditions to obtain the forced response of the microring system.

The amplitude-frequency response is shown in Figure 6 when DC voltage $V_{d}=0.5$, AC voltage amplitude $V_{a}=1$ and damping ratio $\xi=0.001$. The electrode span interval is $[0, \pi / 3]$. Response amplitude changes with electrode span. In addition to the peaks near the natural frequency in the figure, there are also obvious peaks at $\omega_{1} / 2$ and $2 \omega_{1}$. The square of the potential difference can be written $V^{2}=V_{D}^{2}+2 V_{D} V_{A} \sin (\Omega t)+V_{A}^{2}(1-\cos (2 \Omega t)) / 2$. When the voltage frequency is $\Omega$, the electrostatic force has two frequency components $\Omega$ and $2 \Omega$ at the same time, so a peak appears at $\omega_{1} / 2$. Due to the existence of electrostatic force, an electrostatic stiffness matrix $\left[\mathbf{K}^{e}\right]$ is introduced. The electrostatic stiffness couples the generalized coordinates $\alpha_{k}(t)$ and $\beta_{k}(t)$, so that the system has a peak at the non-natural frequency $2 \omega_{1}$.

Figure 7 shows the relationship between the maximum system response and the electrode span. Other parameters are $V_{d}=0.5, \xi=0.001, \phi=\pi / 3$, and $V_{a}=1$. It can be seen that when the electrode span is $[0, \pi / 4]$, the response amplitude increases as the electrode span increases. When the electrode span range is $[\pi / 4, \pi / 3]$, the response amplitude increases slowly, and finally the response amplitude decreases as the electrode span increases. 


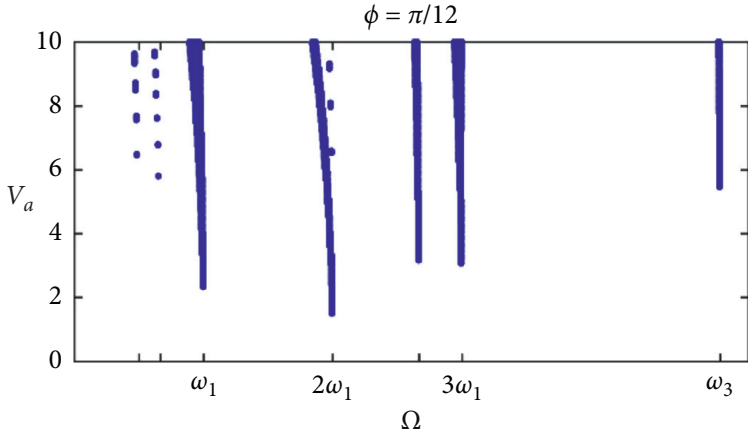

(a)

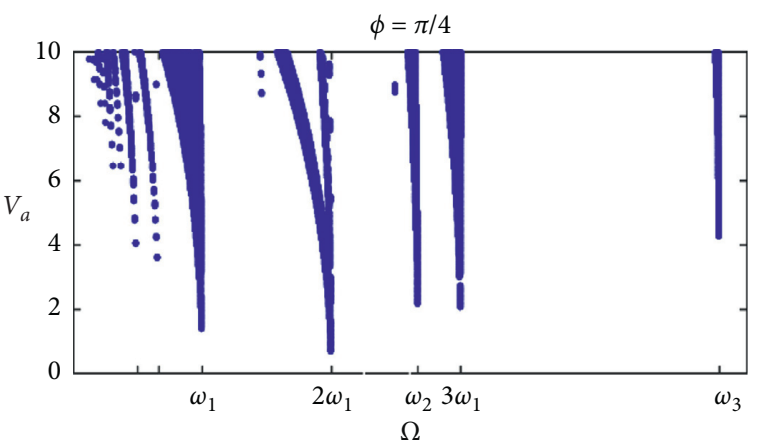

(c)

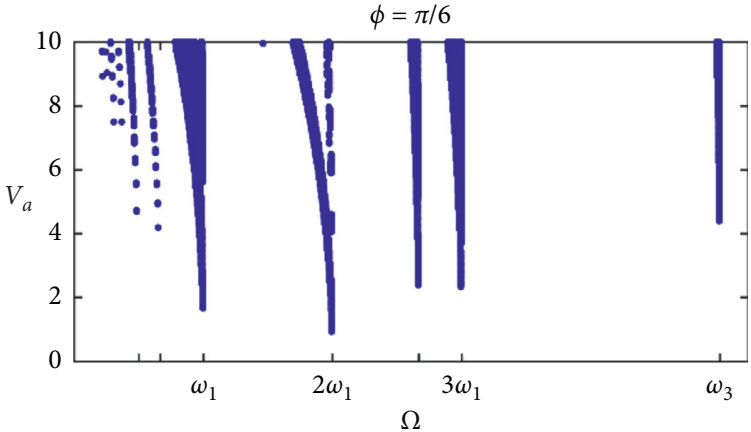

(b)

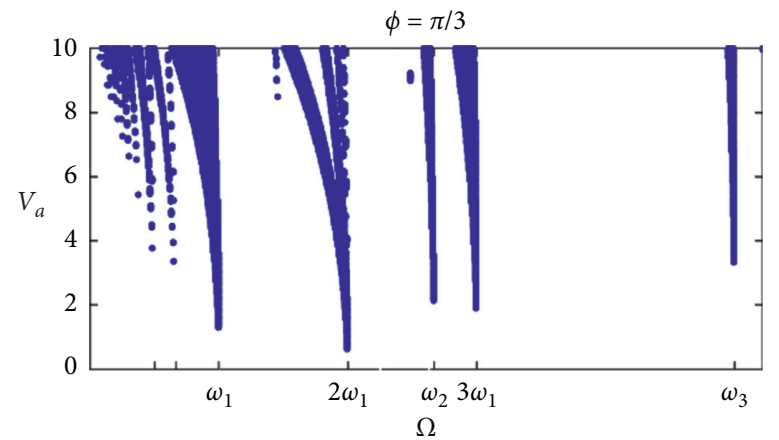

(d)

Figure 4: The unstable region of the system under different electrode spans.

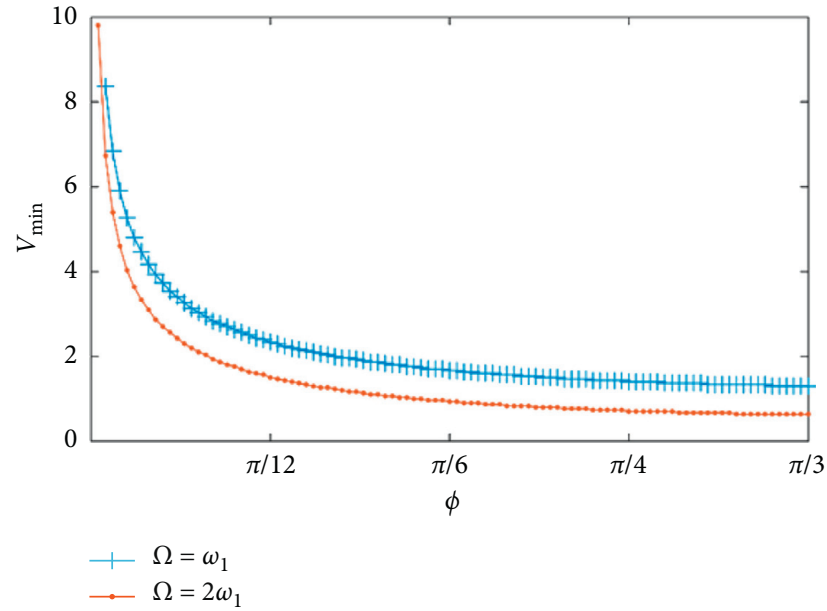

FIgURE 5: The minimum voltage value of unstable region changes with $\phi$. 


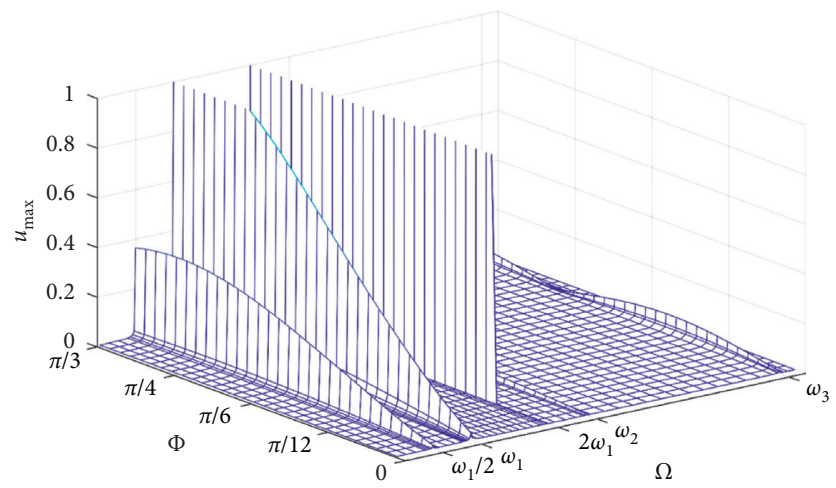

FIgURE 6: Amplitude-frequency response under different electrode spans.

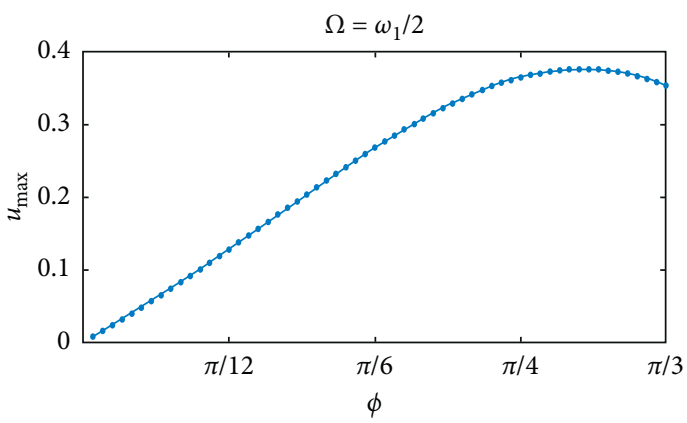

(a)

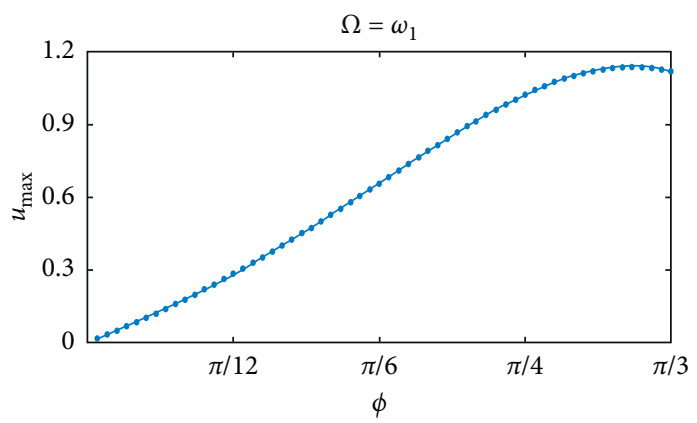

(b)

Figure 7: Response amplitude spectrum of the system at voltage excitation frequency $\omega_{1} / 2$.

\section{Conclusions}

Based on the linearized model of the electrostatic force considering coupling with microring deformation, a dynamic model for a microring system has been established. Due to the coupling effect between the electrostatic force and the deformation of the microring, the stiffness of the microring is related to the electrostatic force parameters. Then, the time-varying voltage makes the stiffness matrix of the system have time-varying characteristics, which in turn causes the dynamic characteristics of the system to become complicated. Though the dynamic model obtained in this paper, the dynamical stability and response of the system with various parameters of the damping and electrode span are analyzed. Some conclusions are summarized as follows:

(1) The unstable region of the system is mainly at the natural frequency and the frequency multiplication. Damping has a greater impact on unstable regions at high frequencies. When the damping is large, the unstable region of the system mainly appears near the first-order natural frequency.

(2) As the electrode span increases, the unstable area of the system increases and the minimum value of the voltage in the unstable region and the rate of change of the minimum voltage value decrease. The response amplitude increases first and then decreases as the increase of the electrode span. These are very helpful for the design of the electrostatically driven microring system.

\section{Data Availability}

All data included in this study are available upon request from the corresponding author.

\section{Conflicts of Interest}

The authors declare no potential conflicts of interest with respect to the research, authorship, and/or publication of this article.

\section{Acknowledgments}

This work was supported in part by the Natural Science Foundation of Shandong Province (ZR2018MEE021), Equipment Pre Research Fund Project (61402100501), and Innovation and Technology Program of Colleges in Shandong Province (2019KJB005).

\section{References}

[1] Y. Huang, A. Sai Sarathi Vasan, R. Doraiswami, M. Osterman, and M. Pecht, "MEMS reliability review," IEEE Transactions on Device and Materials Reliability, vol. 12, no. 2, pp. 482-493, 2012. 
[2] B. J. Gallacher, J. S. Burdess, and K. M. Harish, "A control scheme for a MEMS electrostatic resonant gyroscope excited using combined parametric excitation and harmonic forcing," Journal of Micromechanics and Microengineering, vol. 16, no. 2, pp. 320-331, 2006.

[3] F. T. Han, Q. P. Wu, and L. Wang, "Experimental study of a variable-capacitance micromotor with electrostatic suspension," Journal of Micromechanics and Microengineering, vol. 20, no. 11, p. 115034, 2010.

[4] Y. Ma, S. Islam, and Y.-J. Pan, "Electrostatic torsional micromirror with enhanced tilting angle using active control methods," IEEE/Asme Transactions on Mechatronics, vol. 16, no. 6, pp. 994-1001, 2010.

[5] C. Comi, A. Corigliano, A. Ghisi, and S. Zerbini, "A resonant micro accelerometer based on electrostatic stiffness variation," Meccanica, vol. 48, no. 8, pp. 1893-1900, 2013.

[6] J.-E. Wong, J. H. Lang, and M. A. Schmidt, "An electrostatically-actuated MEMS switch for power applications," in Proceedings of theEEE Thirteenth Annual International Conference on Micro Electro Mechanical Systems (Cat. No. 00CH36308), pp. 633-638, IEEE, Miyazaki, Japan, January 2000.

[7] Ö. Civalek and C. Demir, "A simple mathematical model of microtubules surrounded by an elastic matrix by nonlocal finite element method," Applied Mathematics and Computation, vol. 289, pp. 335-352, 2016.

[8] B. Akgöz and Ö. Civalek, "Effects of thermal and shear deformation on vibration response of functionally graded thick composite microbeams," Composites Part B: Engineering, vol. 129, pp. 77-87, 2017.

[9] F. Ebrahimi, M. R. Barati, and Ö. Civalek, "Application of Chebyshev-Ritz method for static stability and vibration analysis of nonlocal microstructure-dependent nanostructures," Engineering with Computers, vol. 36, no. 3, pp. 953-964, 2019.

[10] Ö. Civalek, B. Uzun, M. Ö. Yaylı, and B. Akgöz, "Size-dependent transverse and longitudinal vibrations of embedded carbon and silica carbide nanotubes by nonlocal finite element method," The European Physical Journal Plus, vol. 135, no. 4, p. $381,2020$.

[11] Ç. Demir and Ö. Civalek, "Torsional and longitudinal frequency and wave response of microtubules based on the nonlocal continuum and nonlocal discrete models," Applied Mathematical Modelling, vol. 37, no. 22, pp. 9355-9367, 2013.

[12] F. M. Alsaleem, M. I. Younis, and H. M. Ouakad, "On the nonlinear resonances and dynamic pull-in of electrostatically actuated resonators," Journal of Micromechanics and Microengineering, vol. 19, no. 4, Article ID 045013, 2009.

[13] J. Han, Q. Zhang, and W. Wang, "Static bifurcation and primary resonance analysis of a MEMS resonator actuated by two symmetrical electrodes," Nonlinear Dynamics, vol. 80, no. 3, pp. 1585-1599, 2015.

[14] J. Machowski, J. Bialek, and J. Bumbypp. 3-5, John Wiley, Hoboken, NJ, USA, 2008.

[15] J. A. Richards, Analysis of Periodically Time-Varying Systems, pp. 50-51, Springer Science \& Business Media, Berlin, Germany, 2012.

[16] F. M. Alsaleem, M. I. Younis, and L. Ruzziconi, “An experimental and theoretical investigation of dynamic pull-in in MEMS resonators actuated electrostatically," Journal of Microelectromechanical Systems, vol. 19, no. 4, pp. 794-806, 2010.

[17] B. Wang, S. Zhou, J. Zhao, and X. Chen, "Size-dependent pullin instability of electrostatically actuated microbeam-based
MEMS," Journal of Micromechanics and Microengineering, vol. 21, no. 2, Article ID 027001, 2011.

[18] H. Farokhi, M. H. Ghayesh, and S. Hussain, "Pull-in characteristics of electrically actuated MEMS arches," Mechanism and Machine Theory, vol. 98, pp. 133-150, 2016.

[19] Y. Mafinejad, A. Kouzani, K. Mafinezhad, and R. Hosseinnezhad, "Low insertion loss and high isolation capacitive RF MEMS switch with low pull-in voltage," The International Journal of Advanced Manufacturing Technology, vol. 93, no. 1-4, pp. 661-670, 2017.

[20] J. Francis and P. A. Michael, "Investigation of micro/nano motors based on renewable energy sources," Materials Today: Proceedings, vol. 27, pp. 150-157, 2020.

[21] L. Yan, D. Liu, H. Lan, and Z. Jiao, "Compact traveling wave micromotor based on shear electromechanical coupling," IEEE/ASME Transactions on Mechatronics, vol. 21, no. 3, pp. 1572-1580, 2016.

[22] L. Ran, "A novel three-dimensional contact model of piezoelectric traveling wave ultrasonic micromotor," Smart Materials and Structures, vol. 29, p. 7, 2020.

[23] M. Bao and H. Yang, "Squeeze film air damping in MEMS," Sensors and Actuators A: Physical, vol. 136, no. 1, pp. 3-27, 2007.

[24] P. Belardinelli, M. Brocchini, L. Demeio, and S. Lenci, "Dynamical characteristics of an electrically actuated microbeam under the effects of squeeze-film and thermoelastic damping," International Journal of Engineering Science, vol. 69, no. aug, pp. 16-32, 2013.

[25] W.-C. Chuang, H.-L. Lee, P.-Z. Chang, and Y.-C. Hu, "Review on the modeling of electrostatic MEMS," Sensors, vol. 10, no. 6, pp. 6149-6171, 2010.

[26] W.-M. Zhang, H. Yan, Z.-K. Peng, and G. Meng, "Electrostatic pull-in instability in MEMS/NEMS: a review," Sensors and Actuators A: Physical, vol. 214, pp. 187-218, 2014.

[27] B. Chouvion, S. McWilliam, and A. A. Popov, "Effect of nonlinear electrostatic forces on the dynamic behaviour of a capacitive ring-based Coriolis Vibrating Gyroscope under severe shock," Mechanical Systems and Signal Processing, vol. 106, pp. 395-412, 2018.

[28] S. Sieberer, S. McWilliam, and A. A. Popov, "Nonlinear electrostatic effects in MEMS ring-based rate sensors under shock excitation," International Journal of Mechanical Sciences, vol. 157-158, pp. 485-497, 2019.

[29] W. Soedel and M. S. Qatu, pp. 64-69, Acoustical Society of America, 2005.

[30] L. Xu and L. Qin, "Nonlinear analysis for a novel actuator," Mechanism and Machine Theory, vol. 45, no. 3, pp. 426-437, 2010.

[31] Y. C. Hu, C. M. Chang, and S. C. Huang, "Some design considerations on the electrostatically actuated microstructures," Sensors and Actuators A: Physical, vol. 112, no. 1, pp. 155-161, 2004.

[32] X. Ye, Y. Chen, D.-C. Chen, K.-Y. Huang, and Y.-C. Hu, "The electromechanical behavior of a microring driven by traveling electrostatic force," Sensors, vol. 12, no. 2, pp. 1170-1180, 2012.

[33] Y. Li, T. Yu, and Y.-C. Hu, "The stiffness variation of a microring driven by a traveling piecewise-electrode," Sensors, vol. 14, no. 9, pp. 17256-17274, 2014.

[34] T. Yu, J. Kou, and Y.-C. Hu, "Vibration of a rotating microring under electrical field based on inextensible approximation," Sensors, vol. 18, no. 7, p. 2044, 2018.

[35] A. Fargas-Marques, J. Casals-Terré, and A. M. Shkel, "Resonant pull-in condition in parallel-plate electrostatic 
actuators," Journal of Microelectromechanical Systems, vol. 16, no. 5, pp. 1044-1053, 2007.

[36] S. C. Huang and W. Soedel, "Effects of coriolis acceleration on the free and forced in-plane vibrations of rotating rings on elastic foundation," Journal of Sound and Vibration, vol. 115, no. 2, pp. 253-274, 1987.

[37] K. Farhang and A. Midha, "Steady-state response of periodically time-varying linear systems, with application to an elastic mechanism," Journal of Mechanical Design, vol. 117, no. 4, pp. 633-639, 1995. 